\title{
Tangence
}

\section{Michel Tournier, Édouard Boubat et Vancouver}

\section{Joëlle Cauville}

Numéro 48, octobre 1995

Montréal et Vancouver : parcours urbains dans la littérature et le cinéma

URI : https://id.erudit.org/iderudit/025867ar

DOI : https://doi.org/10.7202/025867ar

Aller au sommaire du numéro

Éditeur(s)

Tangence

ISSN

0226-9554 (imprimé)

1710-0305 (numérique)

Découvrir la revue

Citer cet article

Cauville, J. (1995). Michel Tournier, Édouard Boubat et Vancouver. Tangence, (48), 128-135. https://doi.org/10.7202/025867ar d'utilisation que vous pouvez consulter en ligne.

https://apropos.erudit.org/fr/usagers/politique-dutilisation/ 


\section{Michel Tournier, Édouard Boubat et Vancouver Joëlle Cauville}

En septembre 1972, grâce à une bourse du Conseil des Arts, Michel Tournier, en compagnie du photographe Édouard Boubat, effectua la traversée du Canada, des Iles de la Madeleine à Vancouver. Il préparait alors un nouveau roman: Les météores dont le thème central est l'espace et dont un chapitre (chapitre XIX), intitulé "Le Phoque de Vancouver", se passe en Colombie-Britannique.

La découverte du Canada n'a pas uniquement alimenté la fiction romanesque de Tournier mais elle a produit également un journal de voyage: Canada, Joumal de voyage (publié par La Presse en 1977) dont une partie, légèrement modifiée a paru dans le chapitre intitulé "L'espace canadien" d'un recueil d'essais et de photographies: Des clefs et des serrures. Images et proses (publié en 1979). C'est sur ces trois sources, qui se recoupent à bien des égards, que se fonde cette analyse de la place de Vancouver dans l'imaginaire tourniérien.

La ville de Vancouver, tout comme celle de Montréal à laquelle Tournier et Boubat accordent moins d'importance, participe d'un mythe: celui de l'espace canadien comme "grenier à rêves, lointain merveilleux" qui s'oppose à la triste réalité de la vieille Europe: "Confinés en cette petite Europe vieillotte et mesquine, nous avons soif de vastes espaces pour déployer les ailes de notre imagination." 1

Avant d'être une réalité, le Canada avait été, pour Tournier, un rêve élaboré par des lectures de jeunesse: les romans d'aventures de James Oliver Curwood et de Jack London; il représentait à ses yeux une espèce d'Hyperborée comparable à l'Eldorado des Espagnols, aux Indes des Britanniques, à la Prusse Orientale des Allemands. Toutefois, ajoute le romancier, la terre canadienne a un alter ego dans l'imaginaire français, un autre lieu mythique: le Sahara: mêmes surfaces immenses $\left(9,3\right.$ millions de $\mathrm{km}^{2}$ canadiens, 7.3 millions de $\mathrm{km}^{2}$ sahariens), des climats extrêmes

1 Michel Tournier, Des clefs et des serrures, Paris, Éditions du Chêne/Hachette, 1979 , p. 141. 
quoique absolument opposés, des noms de six lettres chacun et qui se font écho dans la voyelle $a$ répétée trois fois aux mêmes emplacements. Autant de coïncidences qui font s'interroger Tournier: "Cela fait songer à des notions à la fois complémentaires et antithétiques comme le Yin et le Yang de la pensée chinoise, dont la synthèse est le TAO, principe d'ordre universel. Mais quelle serait la synthèse du Canada et du Sahara? ${ }^{2}$

Pourtant, le Canada, plus que le grand désert africain où se projetèrent Fromentin, Daudet, Maupassant et surtout Gide a, dans l'univers tourniérien, une fonction symbolique: paradisiaque. Il est le paysage d'un certain commencement ou recommencement: "Paradis terrestre, oui, mais non pas ses fleurs, ses fruits, son climat mol et délicieux. Paradis terrestre parce que première terre habitée par le premier homme Terre vierge... "3

Cette conception est proche du pays des Hyperboréens mentionné dans la mythologie grecque, situé dans l'extrême Nord, plus loin que le pays d'où souffle Borée, au-delà du vent du Nord, pays auréolé de rêves, de toutes les enfances et de tous les âges d'or ${ }^{4}$. À l'Adam de La Bible "déjà encombré d'un père autoritaire, Jehovah, d'un oncle séduisant mais dangereux, Le Serpent, d'une épouse geignarde..." 5 , Tournier préfère le Canadien primitif, le trappeur ingénieux et athlétique, subvenant seul à tous ses besoins avec sa cabane en rondins, ses pièges et sa poêle à frire. Il lui sait gré d'avoir envoyé promener la civilisation, "ses flics et ses curés" (ibid., p. 143) et d'avoir pris le premier bateau pour Montréal.

Cette vision d'un Canada paradisiaque, Tournier l'avait déjà prêtée à Tiffauges, le héros du Roi des Aulnes son second roman publié en 1970. Ce dernier, après avoir recréé un Eden canadien en Prusse Orientale où il est captif du régime hitlérien, le voit malheureusement se métamorphoser selon les règles de la grande inversion maligne qui domine tout l'ouvrage. La province de son rêve personnel avec ses forêts et ses orignaux, devient "le trésor d'Auschwitz", comme le lui explique le petit juif Ephraïm, rescapé des camps de la mort:

2 Ibid., p. 143.

3 Michel Tournier, Canada, Joumal de voyage, Montréal, La Presse, 1977, p. 9.

4 Jean Chevalier et Alain Gheerbrant, Dictionnaire des symboles, Paris, Robert Laffont/Jupiter, coll, "Bouquins", 1982, p. 515-516.

5 Michel Tournier, Des clefs et des serrures, op. cit., p. 142. 
130

Tu comprends, les détenus portaient sur eux ce qui leur restait de plus précieux, des pierres fines, des pièces d'or, des bijoux, des montres. Quand on les avait gazés, leurs vêtements étaient rangés avec tout ce qu'on avait trouvé dans leurs poches et les doublures dans un baraquement spécial qu'on appelait justement le Canada... ${ }^{6}$

Et comme pour excuser et justifier cette appellation devant un Tiffauges atterré, il ajoute: "Ah, parce que pour nous, le Canada, c'est la richesse, c'est le bonheur, c'est la liberté. "7 Lorsque Boubat et Tournier entreprennent leur voyage, ils attendent donc du Canada réel qu'il soit le réceptacle de leur œuvre photographique et scripturale, qu'il crée le climat qui permette au Canada mythique, imaginaire d'éclore: "Il y a deux Canada: celui où nous allons et l'autre, celui que nous portons dans notre coeur, le principal. " 8

Les deux compagnons partent en quelque sorte à la recherche du Graal. Leur quête ne s'encombre pas de pittoresque, elle est d'ordre spirituel. D'ailleurs, l'exergue de Canada. Journal de voyage, reprise de la devise canadienne incitant à la solidarité pancanadienne, annonce une démarche initiatique: "Partout au Canada, vous découvrirez une part de vous-même (Explore a part of Canada, and you'll discover a part of yourself.)"

Quelle place occupe Vancouver dans cette aventure? L'imaginaire tournérien rejoint, à ce sujet, le point de vue canadien, à l'est du pays: "Vancouver fait partie des lieux magiques, auréolés de prestige dans mon imagination ${ }^{9}$ et "... D'autant plus que tout le monde s'accorde - sans y être allé - à juger Vancouver comme une ville fascinante, magique, etc. "10

L'anticipation n'est pas la même à l'endroit de Montréal. Dans Les météores, Paul, à la recherche de son jumeau fuyard Jean, souligne le caractère froid de la ville québécoise: selon lui, le vide des Prairies qu'il vient de traverser, effectuant ainsi un voyage en sens inverse de celui de Tournier et Boubat, n'est pas absent de ses larges rues, le Saint-Laurent est trop puissant; le

6 Michel Tournier, Le roi des Aulnes, Paris, Gallimard, 1970 et coll. "Folio", n०656, 1970, p. 556.

7 Ibid.

8 Michel Tournier, Canada, Journal de voyage, op. cit., p. 18.

9 Ibid., p. 76.

$10 \quad$ Ibid., p. 53. 
besson dépareillé déplore le manque de chaleur humaine, de contact animal, de promiscuité des races, inexplicable dans cette énorme cité. Comme pour son créateur, Tournier dans le Joumal de voyage, Montréal est une ville électrique à laquelle le grand jour sied mal. Boubat ne fera aucune photo de la métropole québécoise. Tournier et lui sont gagnés par "la tristesse écrasante [des] gratte-ciel de marbre noir et de verre fumé qui dominent de leur masse funèbre des bâtiments de formes et de dimensions humaines"11. Ainsi, sur la Place d'Armes, le monument à Maisonneuve et l'église Notre-Dame sont-ils écrasés par une banque comme par une dalle funéraire. Seul, le baroque canadien de l'intérieur en bois de l'église Notre-Dame attire leur attention et Tournier de conclure: "Montréal, c'est New York moins la vie intense et le mélange des races qui donnent chaleur et couleurs à la grande cité américaine. " 12

Sous le regard tourniérien, Vancouver est au contraire en tous points comparable à la métropole newyorkaise. La capitale du Far West, tant vantée et si peu connue, a d'abord frappé Tournier comme une authentique ville internationale, plus puissante et plus sérieuse que tout ce qu'il a vu à l'Est, y compris Montréal, et ce grâce au crasseux et au sordide sans quoi il n'y a pas de grande cité. Le héros des Météores, Paul, la trouve même plus colorée, plus vivante, plus hétéroclite que Tokyo. Comme le romancier, il en note l'aspect moins noble mais indispensable à son rang, à savoir les films érotiques, les bars louches, les silhouettes furtives, les débris boueux sur les trottoirs. Dans son journal, Tournier évoque le Vancouver nocturne: "Vancouver by night. Comme ces champignons qui ne poussent que la nuit, le sexe attend que les honnêtes gens soient au lit pour dresser dans les rues sa petite tête de reptile sans yeux. "13

Le second élément spécifique à Vancouver, selon Tournier, est son mélange de races, autre point commun avec les grandes villes américaines : les Japonais en transit, qui se repèrent par "un je ne sais quoi d'étranger et de provisoire" 14, les Indiens: "de jeunes momies qui flottent dans des vêtements trop grands et dont les petits yeux vifs et noirs luisent sous le large rebord du

11 Ibid., p. 69.

12 Ibid.

13 Ibid., p. 102.

14 Michel Tournier, Les météores, op. cit., p. 545. 
132

chapeau ${ }^{15}$," les Chinois, qui constituent la variété la plus nombreuse et vivent dans le quartier fermé de Chinatown, et enfin les Esquimos ${ }^{16}$. Ces races y sont assez nombreuses pour que l'on fasse de Vancouver une ville en partie jaune. Cette remarque donne à Tournier l'occasion de se lancer dans une leçon naturaliste digne de celles du Musée de l'homme, sur la distinction physique entre ces différents peuples. Ainsi, l'Eskimo se reconnait-il par ses joues énormes, son crâne de forme ovale (dolichocéphale) alors que les autres "jaunes" (sic) ont le crâne rond (brachycéphale). Faut-il vraiment accorder une valeur scientifique à ses dires lorsqu'il affirme que le même Eskimo possède, du front à la nuque, une espèce d'arête longitudinale de renforcement "assez semblable à la carène des anciens casques de guerre français" ${ }^{17}$ ? Il lui confère également une rusticité qu'il oppose à la finesse chinoise. Sa graisse, enfin, est différente de celle du blanc: "Dans ce pays d'obèses [le Canada], la graisse de l'Eskimo ne ressemble pas à celle des Blancs. On voit bien que celle-ci est une graisse de pâtisserie et d'ice-cream. La graisse de l'Eskimo sent le poisson et la viande fumée. " 18

Tournier ne peut évoquer Vancouver en omettant son climat: au Canada oriental hémiplégique (les six mois de terrible hiver) succèdent la pluie abondante, l'explosion du soleil après la tempête, l'étrange mariage de la mer et de la forêt à Stanley Park, que Paul, le jumeau des Météores, confie n'avoir rencontré nulle part ailleurs. Il fait aussi référence à la faune, à la familiarité des mouettes:

Çà et là, on en voit une [mouette] très grosse, très sale, perchée sur une murette ou au sommet d'un poteau, l'air renfrogné. On peut s'approcher sans qu'elle bronche. Si on tend la main vers elle, elle envoie en avant son grand bec crochu, soit dans l'espoir d'un bon morceau, soit pour vous rappeler au respect des distances. ${ }^{19}$

et à l'apparition d'un phoque aux yeux bridés, rivé à un rocher à 200 mètres de la plage, comme fasciné par la ville et reflétant l'attitude de ses habitants et de ses visiteurs envoûtés. Urs Kraus,

15 Ibid., p. 545.

16 Michel Tournier utilise ce terme au lieu de celui plus communément accepté d'Inuit.

17 Michel Tournier, Canada, Journal de voyage, op. cit., p. 84.

18 Ibid.

19 Ibid., p. 85. 
le peintre allemand des Météores, s'identifie à l'animal marin: comme lui, il est subjugué par la ville "fraîche et morte à la fois ". 20

La beauté dramatique du site de Vancouver n'est pas dénuée d'ambiguité : en effet, si la ville est un tremplin pour les Orientaux, si elle représente une possibilité de renaissance pour le Japonais, elle ne constitue qu'un aboutissement pour l'Occidental. Vancouver n'est pas pour lui une ville d'initiation comme le seraient Paris, Londres, New York. Le détroit de Georgia y est une eau morte, fermée, à l'horizon bouché par l'Ile de Vancouver; aucun souffle de vent vivifiant ne l'atteint, aucune invitation à l'embarquement, à la découverte du Pacifique. Pour lui, Vancouver est le terminus naturel d'une longue migration est-ouest qui, partie de l'Europe, a traversé l'océan Atlantique et le continent nord-américain.

Urs est partagé entre sa fascination pour la ville et son inquiétude: il avoue sa stérilité sur le plan artistique, son impuissance à créer dans le paradis vancouvérois :

Moi, j'avais eu tout de suite le coup de foudre pour Vancouver, cette ville où des journées entières de pluie préparent les couchers de soleil les plus sublimes. Impossible de peindre, ici! L'ivresse de l'impuissance! La toile reste vierge pendant qu'on s'en met plein la vue! C'était une drogue dont je voulais épuiser les pouvoirs avant de continuer. ${ }^{21}$

Le journal de voyage comme le roman présente la même vision apocalyptique de Vancouver:

...Le rideau de nuages noirs se déchire, s'effiloche, forme des masses échevelées entre lesquelles s'ouvrent des brèches bleues où le soleil explose. Les grands arbres mouillés du Stanley Park s'ébrouent dans le vent et leur odeur forestière et moussue heurte les remugles de vase et de varech qui montent de la grève... ${ }^{22}$

et: "Le ciel n'est qu'une lumineuse dévastation, forteresses de vapeur croulantes, citadelles de lumière au pillage, chevauchées furieuses d'escadrons neigeux. "23

20 Michel Toumier, Les métēores, op. cit., p. 547.

21 Ibid., p. 553.

22 Ibid., p. 547.

23 Michel Tournier, Canada, Journal de voyage, op. cit., p. 90. 
134

En plus de refléter un univers ambigu, la ville de Vancouver cristallise, plus que toute autre ville canadienne, une réflexion sur la vacuité de l'espace canadien que Tournier oppose à l'espace plein japonais où l'on est obsédé par la peur de l'étouffement. Au contraire, le Canadien "titube de vertige " ${ }^{24}$ dans des espaces immenses qu'il cherche vainement à peupler. Tandis que le Japonais invente des structures propres à donner un statut indéfini à des espaces très réduits - le jardin zen, par exemple, crée un vide abstrait où la pensée peut se déployer - les Canadiens obéissent à un mouvement inverse de repliement: "...un mouvement de retrait, de recroquevillement, voire d'inhumation (galeries et passages souterrains) pour lutter contre le vide, l'immensité du pays. " 25

Les photos d'Edouard Boubat prises à Vancouver illustrent avec bonheur l'un des traits les plus constants de la psychologie canadienne: une certaine façon de dire non à l'espace extérieur. Ainsi, cette vue des immeubles du centre ville, peuplèe par la seule minuscule silhouette d'un sportif courageux; ou encore ce jeune homme endormi, blotti dans une des grosses souches ensablées qui tiennent lieu de rochers sur la grève de Vancouver; ou cette même plage, totalement déserte avec sa façade irréelle de gratte-ciel apparemment inhabités.

Selon Michel Tournier, le gratte-ciel, loin de répondre à un manque d'espace, est la réaction normale à l'excès d'espace, à l'angoisse de l'ouverture. La tour domine et maîtrise l'espace qui la cerne et elle devient un centre de ralliement pour les hommes dispersés: "Elle est centrifuge pour qui l'habite, centripète pour ceux qui sont éparpillés à la périphérie. " 26 C'est à Vancouver, à travers les propos du peintre philosophe Urs, que Paul, le jumeau des Météores, apprend une importante leçon sur le rôle de l'espace dans notre vie. Selon Urs, dont le point de vue est tout à fait bergsonien, l'espace devient une métaphore du temps; la situation d'un objet dans l'espace, loin d'être indifférente, met en cause sa nature même:

Bref, il n'y a pas de translation sans altération. C'est la négation de la géométrie, de la physique, de la mécanique qui toutes

24 Michel Tournier, Des clefs et des serrures, op. cit., p. 137.

25 Ibid., p. 138.

26 Michel Tournier, Canada, Joumal de Voyage, op. cit., p. 114. 
supposent comme condition première un espace vide et indifférencié où tous les mouvements, déplacements et permutations sont possibles sans changement substantiel pour les mobiles qui y évoluent. ${ }^{27}$

Les différents portraits qu'Urs a exécutés de Jean, le jumeau de Paul, onze au total, ne ressemblent plus à son frère, en raison de leur position différente dans l'espace. Paul prend ainsi conscience que son frère n'est plus l'objet de sa quête, que son voyage a changé de sens: son histoire n'aura été qu'une longue et aventureuse méditation sur la notion d'espace. À la fin du roman, amputé de son côté gauche broyé dans le tunnel de Berlin, ville symbolique associée au déchirement de la cellule gémellaire, il se souviendra de la leçon de Vancouver sur l'espace-qualité: le jumeau survivant est désormais le seul tenant de l'espace intergémellaire - l'âme déployée - et il absorbe son frère fuyard disparu: "grâce à ce grand voyage dans l'espace-qualité, grâce à l'altération-initiation subie jusqu'au sang, grâce à la mainmise de l'homme chronologie sur le ciel météorologique. " 28

Ainsi, au-delà du propos anecdotique et pittoresque auquel Tournier, disciple du naturalisme, n'a pas pu s'empêcher de succomber, la ville de Vancouver jouit, sur un plan symbolique et philosophique, d'un rôle de premier plan dans l'univers tourniérien. Dans Les météores, au même titre que Venise, elle contribue à l'évolution de la quête de Paul en étant, après l'expérience japonaise, une étape de sa réflexion sur l'espace qu'il continuera à travers les Prairies canadiennes jusqu'à son retour tragique à Berlin. Bien que l'écrivain répète que la métropole de la Colombie-Britannique n'est pas une ville d'initiation pour l'occidental, il lui confère ce rôle dans la vie de son héros qui, comme un Japonais, traverse le Canada d'ouest en est.

27 Michel Tournier, Les météores, op. cit., p. 54.

28 Tournier, Michel, Le vent paraclet, Paris, Gallimard, coll. "Folio 1138, 1977 p. 278. 